\title{
LA ENSEÑANZA DE LOS MODELOS SOBRE LA NATURALEZA DE LA LUZ
}

\author{
BELÉNDEZ, A. ${ }^{(1,3)}$ PASCUAL, I. (2), y ROSADO, L..$^{(3)}$
}

(1) Departamento de Física Aplicada (Sección Alicante), E.U. Politécnica de Alicante (Universidad Politécnica de Valencia), Alicante.

(2) Departamento Interuniversitario de Óptica, E.U. de Óptica (Universidad de Alicante). Alicante.

(3) Facultad de Ciencias y Programa de Formación del Profesorado, UNED Madrid.

\section{SUMMARY}

Contemporary ideas on science learning is useful to avoid the failure of traditional teaching related to preconcepts or conceptual errors in both students and teachers. In this context the student is introduced to the study of light through the knowledge of the different models or theories which explain the phenomena of light. The different teaching levels are born in mind so as to follow a process of both conceptual and methodological change.

\section{INTRODUCCIÓN Y PLANTEAMIENTO GENERAL}

La intención última de Ia Ciencia consiste en la explicación de los hechos de la Naturaleza. Para ello, la Ciencia se ocupa de observar y recoger los datos que tales hechos proporcionan y de ordenarlos de tal manera que sus compendios ordenados nos permitan conocer mejor la Naturaleza. Esta idea de resumir y concentrar nuestro conocimiento acerca del mundo que nos rodea es lo que nos lleva a la elaboración de teorías (Morales 1987).

Las teorías se desarrollan en torno a uno o varios conceptos fundamentales. EI primer requisito para comprender los fenomenos es, por tanto, introducir los conceptos adecuados. Sin la ayuda de los conceptos válidos no podemos saber realmente qué ha sido observado. Al intentar analizar nuevos fenómenos siempre es necesario introducir conceptos nuevos que, por lo general, se presentan en forma poco clara y desarrollada. Luego son modificados, o abandonados, o sustituidos por otros mejores que quedan más claramente definidos aunque nunca se puede asegurar su perennidad (Meheut y Larcher 1987).

En relación a esta discusión concluimos que la Física no es una mera yuxtaposición de descubrimientos y observaciones experimentales, a la cual se agregue su descripción matemática. Es también historia de los conceptos (Heisenberg 1979).

Por tanto, la Física, al describir mediante modelos la Naturaleza, posee ciertas peculiaridades que influyen decisivamente en los métodos a seguir en su aprendizaje. Éste debe ser un aprendizaje de conceptos y teorías

físicas (o sea, de modelos). Además tiene que contemplar una evolución de estos conceptos y teorías, enmarcándolos en la historia de la Física e indicando sus rangos de validez, los fenomenos que son capaces de explicar y, a la vez, haciendo hincapié en el hecho de que las teorías son "representaciones" de la Naturaleza pero no la Naturaleza misma. Es decir, puede concebirse el aprendizaje de la Física como cambio conceptual y metodológico (Gil y Carrascosa 1985, Furió y Hernández 1985), teniendo en cuenta tanto las estructuras conceptuales previas de los alumnos como sus tendencias metodologicas a sacar conclusiones precipitadas a partir de observaciones cualitativas.

La dificultad que encuentran muchas veces los alumnos al presentárseles las teorías que explican los fenómenos físicos (en algunas ocasiones radicalmente opuestas a las ya conocidas y asimiladas por los mismos), se debe a una falta de capacidad para exponer a los alumnos la idea de "teoría física". Se entiende por esta expresión un sistema de conceptos, definiciones y leyes que permiten sin contradicciones describir y representar matemáticamente un extenso número de fenómenos (Scheider 1986). Las teorías físicas se derivan de los hechos empíricos mediante la observación y la experimentación (Heisenberg 1979, Morales 1987), y una característica importante de éstas es su capacidad para explicar y al mismo tiempo predecir. Por tanto, no debe descuidarse en el aprendizaje de la Física su componente experimental. En muchas ocasiones, y por la comodidad que ello supone, se hace énfasis en la representación matemática de las leyes físicas (Hickman 1986, Wenhan et al. 1972), olvidando describir los con- 
ceptos básicos y las leyes mismas, y viceversa. La Mecánica Newtoniana es en este sentido un prototipo de teoría física. Otros ejemplos son la Termodinámica, la Mecánica Relativista, la Teorfa Electromagnética de Maxwell, la Mecánica Cuántica y, en relación al tema que nos ocupa, las distintas teorías sobre la luz: la Óptica Geométrica, la Óptica Ondulatoria y la Óptica Cuántica.

Una tarea del profesor es hacer comprender al alumno que las distintas teorías físicas poseen un campo de aplicación acotado, cuyas fronteras vienen dadas, en lo esencial, por los conceptos empleados en cada una de ellas. Fuera de este terreno no puede representar la teoría los fenómenos naturales, porque sus conceptos no son capaces de aprenderlos. Así, la Optica Geométrica mediante los conceptos de rayo luminoso e índice de refracción explica la reflexión en un espejo o las imágenes formadas por las lèntes, pero no es capaz de interpretar los fenómenos de interferencia y difracción de la luz, que necesitan de la Óptica Ondulatoria y del concepto de onda para caracterizar la luz, lo que además la dota de una entidad física, frente a la Óptica Geométrica que no es más que una pura geometría. Por último, el efecto fotoeléctrico o el Compton no tienen cabida dentro de un modelo ondulatorio para la luz, y encuentran explicaciones en la Óptica Cuántica con el uso del concepto de fotón, concepto que, a su vez, ha pasado por muchas y diversas acepciones.

Finalmente indiquemos que las concepciones y modelizaciones utilizadas en la enseñanza de la Óptica, tanto en las clases como en los libros de tex to (rayo luminoso, frente de onda, onda electromagnética, energía cuantizada, fotón, etc.), no suelen ser presentadas como modelos, es decir, representaciones constituidas, "calculables", más o menos simplificadas, ni incluso representaciones, sino como la realidad directamente visible. En estas condiciones, es fácil comprender las dificultades y fracasos de los alumnos para relacionar las teorías como construcciones hipotéticas, sino que las asimilan como dogmas definitivos y cerrados (Martinand 1986, Solbe et al. 1987).

\section{UNA RESPUESTA PARA LA ENSEÑAN- ZA DE LAS TEORIAS DE LA LUZ}

Consideramos la programación en la enseñanza de las teorías de la luz como cambio conceptual según los niveles eđucativos: elemental (9-13 años), medio (1418 años) y superior (18-23 años), vtilizando modelos dentro de cada una de las teorías y según el nivel de que se trate. Desde un punto de vista pedagógico es importante utilizar modelos, y por las mismas razones por las que los utilizan los físicos. Los modelos posibles al analizar los hechos físicos son los siguientes (Rosado 1986): modelos icónicos o a escala (descripción detallista mediante maquetas del objeto al que se refiere), modelos gráficos, modelos matemáticos (formulación matemática que permite describir situaciones físicas y hacer predicciones), modelos conceptuales (referencia a imágenes conceptuales, en ocasiones una representa- ción figurativa) y el modelo científico (modelo conceptual + leyes físicas), este último es el que corresponde propiamente a una teoría. Es importante eliminar problemas tradicionales, como los que aparecen de una transición discontinua de la enseñanza entre dos niveles educativos consecutivos. Para minimizar estos problemas se requiere de un esfuerzo de adaptación por parte del alumno, así como suprimir la descoordinación generalizada entre la formación y los conocimientos adquiridos en una etapa, y la formación y conocimientos básicos requeridos en la nueva fase que comienza.

La programación que se propone considera los conceptos y leyes más importantes que el alumno debe conocer y asimilar, presentando en cada nivel unos objetivos generales y específicos (Rosado 1979), una selección de contenidos y modelos, y por último, unas ideas acerca de los recursos diđácticos a utilizar para alcanzar los objetivos propuestos. Sin embargo, en este trabajo no se pretende hacer un desarrollo detallado de la programación de los contenidos en las distintas etapas de la enseñanza, sino simplemente introducir unas ideas generales sobre dicha enseñanza.

\subsection{Nivel elemental}

En el nivel elemental, a partir de los fenómenos de refracción y reflexión de la luz se introducen los conceptos de rayo Iuminoso (relacionado con la luz) y de índice de refracción (relacionado con los medios materiales); por tanto sólo se considera la Óptica Geométrica (el modelo científico).

Como objetivo pueden proponerse que: conozca que la luz se propaga en línea recta (concepto de rayo luminoso), que observe la desviación de los "rayos luminosos" al pasar la luz de un medio a otro (concepto de índice de refracción), que distinga los distintos tipos de lentes, que comprenda el fenómeno de la dispersión de la luz por prismas, etc. Los contenidos deben ser sencilios y claramente visualizados en la propia clase o en pequeñas experiencias de laboratorio, de modo que el alumno aprenda a observar e incluso a inferir hipótesis. Los modelos a utilizar deben ser los icónicos y analógicos, y los recursos didácticos pueden completarse con algunas experiencias que el alumno pueda realizar por sí mismo. Para introducir las leyes de la refracción y reflexión y los conceptos antes señalados debe partirse siempre de hechos experimentales, de modo que luego se pueda deducir la formación de imágenes a través de lentes y espejos mediante modelos gráficos (construcción gráfica de la imagen).

En este nivel no se tratarán las teorías ondulatoria y cuántica.

\subsection{Nivel medio}

Siguiendo con el mismo planteamiento del nivel elemental, Ios conceptos para la Óptica Geométrica son los mismos. Ahora se utilizarán además modelos mate- 
máticos sencillos (lo que se conoce como aproximación paraxial): expresión matemática de la refracción y reflexión, fórmulas de las lentes, etc. Ya es interesante indicar que la Óptica Geométrica es una teoría más sobre la luz, pero no la única, especificando sus campos de aplicación sobre todo en el diseño y fabricación de instrumentos ópticos (anteojos, telescopios, cámaras fotográficas...).

La teoría ondulatoria de la luz (modelo científico) puede presentarse a partir de modelos analógicos para explicar fenómenos como las interferencias, usando una cubeta de ondas. Para el alumno, el concepto de onda es difícil de comprender y puede utilizarse el modelo conceptual de onda sinusoidal, con crestas y valles, e introducir otros conceptos como la longitud de onda, indicando su variación según el color. Disponer de un pequeño láser sería de gran utilidad para mostrar tanto fenómenos de la Óptica Geométrica (trayectorias rectilíneas) como Ondulatoria (interferencias y difracción), con lo que se consigue contrastar estas dos teorías que parten de conceptos totalmente diferentes.

En los últimos cursos del nivel medio puede empezar a hablarse del concepto de fotón y de la nueva teoría de la luz relacionada con el mismo (Teoría Cuántica de la luz -el modelo científico-). Se debe concluir con una comparación de los tres modelos, contrastando sus conceptos de partida y sus campos de aplicación, con el uso de bastantes ejemplos. Puede hablarse de un doble comportamiento de la luz: ondulatorio en su propagación y corpuscular en su interacción con la materia. Así pues, en este nivel debe introducirse la teoría ondulatoria de la luz, y profundizarse en ella con el modelo matemático de la misma, relacionarla con la teoría geométrica y abrir el camino para el modelo cuántico, comparando éste con los modelos atómicos y la dualidad onda-corpúsculo. Aparecen dificultades en el aprendizaje del modelo cuántico si no se realiza ese aprendizaje como cambio conceptual, partiendo de la propia evolución histórica de las teorías ondulatoria y corpuscular (Gil, Senent y Solbes 1987).

\subsection{Nivel superior}

En el nivel superior debe completarse todo lo ya conocido de los niveles anteriores, sobre todo con modelos matemáticos (Hickman 1986) bien asentados sobre los conceptos y la experiencia. Es importante que el alumno piense detenidamente en lo que en realidad es la luz, sobre la cual existen distintas teorías que, aunque basadas en conceptos y principios bien diferenciados, nos permiten saber cómo se comporta la luz y hacer predicciones según las características del fenómeno analizado. Es decir, la luz manifiesta uno tu otro comportamiento dependiendo del fenómeno óptico que se estudie. Es importante matizar las últimas aplicaciones tecnológicas de la Óptica, así como las aplicaciones de la Óptica en otras Ciencias, de manera especial en las que tienen relación con la carrera que el alumno esté cursando. Las experiencias de laboratorio y la resolución de ejercicios prácticos son fundamentales, $y$ han de guardar una completa relación con el modelo matemático presentado. Hay que intentar huir de la imagen incorrecta, que en muchas ocasiones produce el excesivo peso del empirismo o del formalismo, indicando claramente las rupturas científicas existentes frente a una imagen lineal y acumulativa del desarrollo científico.

\subsection{Proceso básico de enseñanza-aprendizaje}

El proceso de enseñanza-aprendizaje consistirá, en todos los casos, en una presentación del fenómeno en el cual interviene la Iuz, junto con las leyes experimentales que gobiernan dicho fenómeno. Paralelamente se introducirán los conceptos necesarios (apoyados en definiciones claras). A partir de aquí, y teniendo en cuenta las hipóstesis simplificadoras que siempre se hacen, se llega a la formulación del modelo o teoría final. Este modelo servirá, por sí mismo, para dar una explicación de otros fenómenos experimentales no considerados al inicio e incluso para hacer predicciones.

Es interesante comparar las distintas teorías necesarias para interpretar los diferentes fenómenos Iuminosos, de modo que cada teoría tenga un proceso de enseñanza como sigue:

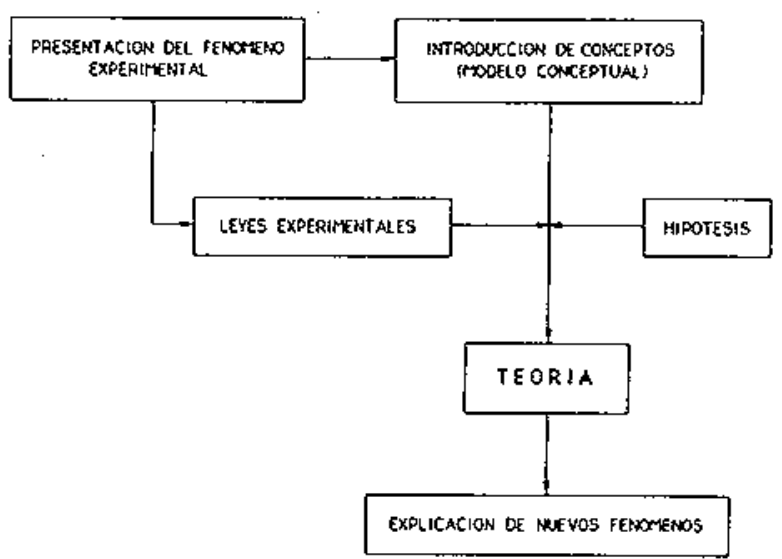

Como puede verse se trata simplemente de la utilización y adaptación del método científico (Chalmers 1984) al proceso de aprendizaje de las Ciencias Experimentales.

\section{CONCLUSIONES}

La enseñanza de la Óptica, y en generai la introducción de los modelos sobre la naturaleza de la luz, es quizás uno de los apartados más descuidados del desarrollo curricular desde el nivel elemental hasta los primeros años de Universidad, a consecuencia, sobre todo, de la poca interconexión que existe entre las enseñanzas de 
los distintos cursos. En éstos no hay un planteamiento lógico de la introducción de los conceptos físicos y su evolución, que el alumno debe y puede asimilar en función de su edad. Junto a ello, el contenido predominante de la Mecánica y la Electricidad que se encuentra en todos los programas y libros de texto (en los niveles elemental y medio), impide un mejor conocimiento de otras partes de la Física como es la correspondiente al estudio de los fenómenos en los que interviene la luz. A esto hay que añadir la falta de unión entre los conceptos y modelos que se explican sobre la luz, de modo que es difícil que los alumnos puedan relacionar y contrastar Ias ideas aprendidas de Óptica Geométrica, Óptica Ondulatoria y, sobre todo, Ias ideas cuánticas acerca de la naturaleza de la luz.

Nos encontramos, por tanto, ante un tema deI cual se ha escrito, investigado y discutido mucho desde los comienzos de la Física; sin embargo, apenas es tratado en las programaciones de Física de los niveles no universitarios. Esto hace que los alumnos acumulen una gran cantidad de errores conceptuales a lo largo de sus años de estudio y cuya eliminación es tarea difícil de realizar. Sumado a esto están muchas veces los errores, también conceptuales, que los propios profesores tienen, debido a un insuficiente conocimiento global de la Didáctica de la Física y del aprendizaje de la Óptica en particular, y originados por la propia enseñanza recibida.

Sería interesante plantear un desarrollo curricular lógico y equilibrado. Introducir los distintos conceptos en función de la edad de modo que los alumnos puedan asimilarlos y no escandalizarse de los distintos modelos que sobre la naturaleza de la luz se han desarrollado. Llegar a comprender sus limitaciones, entendiendo que no unos son completamente falsos ni otros completamente verdaderos. A todo esto hay que añadir el creciente papel de la Óptica en la Ciencia y Tecnología modernas (Bernabeu 1985). El desarrollo del láser, las fibras ópticas, la comunicación por fibras, la óptica integrada, Ia holografía, etc. (Rosado 1987), hace necesario que el joven de hoy esté informado sobre los aspectos fundamentales de la luz, su naturaleza o naturalezas y sus aplicaciones.

En definitiva, en una programación lógica los aspectos geométricos, ondulatorios y corpusculares de la Iuz han de ser tratados con mayor o menor profundidad, pero siempre de modo que se vayan asentando poco a poco, desde el colegio hasta los años de Universidad. En los libros de texto hay que dar a la Óptica la importancia que se merece, dejando de ser las distintas asignaturas de Física sólo enseñanzas de Mecánica y Electricidad, en detrimento de aquélla. Además, y como novedad, sería interesante tratar la Óptica lo antes posible (Rosado 1979), contrariamente a lo que sucede en los programas tradicionales antes mencionados, y esto puede justificar, dado que el niño se relaciona muy pronto con la Iuz, los espejos y las lentes. Unido a esto, para desarrollar las principales nociones de Óptica sólo se requiere la aplicación de expresiones geométricas sencillas. Por último, la Óptica permite que el alumno analice directamente una evolución y cambio de conceptos en esta parte de la Física, y el conocimiento de distintas teorías, lo que luego podrá extrapolar fácilmente a la Mecánica, la Electricidad y la Física Atómica. Desde un punto de vista pedagógico, la enseñanza y experimentación en Óptica constituye una herramienta sumamente útil en la comprensión de los fenómenos físicos.

Finalmente, la Óptica presenta una ventaja adicional: los distintos modelos sobre la misma se estudian desde el nivel elemental, lo que permite su análisis y evolución. Esto no ocurre, por ejemplo, con las teorías de la Mecánica, pues sólo en cursos avanzados de la Universidad se presentan otras teorías a parte de la Newtoniana, como son la Relativista y la Cuántica. Mientras la primera es estudiada durante todos los cursos de los niveles elemental, medio y hasta superior, las dos últimas sólo son tratadas en el nivel superior, lo que no permite, en este caso, un aprendizaje mediante un verdadero cambio conceptual.
REFERENCIAS BIBLIOGRÁFICAS

BERNABEU, E., 1985. La Óptica y las nuevas tecnologias, Prospectiva de la enseñanza de la Optica en España, Vol. I, pp. 37-48.

CHALMERS, A., 1984. ¿Qué es esa cosa llamada Ciencia? (Siglo XXI Editores: Madrid).
FURIÓ, C. y HERNÁNDEZ, J,, 1985. La enseñanza de la teoría atómica como cambio conceptual y metodológico, Enseñanza de las Ciencias. Número Extra, p. 60.

GIL, D. y CARRASCOSA, J., 1985. Science Learning as a conceptual and methodological change, European Journal of Science Education, 7, pp. 231-236. 
GIL, D., SENENT, F. y SOLBES, J, 1987. La introduccion a la Física Moderna: un ejemplo paradigmático de cambio conceptual, Enseñanza de las Ciencias, Número Extra, pp. 209-210.

HEISENBERG, W., 1979. Encuentros con Einstein y otros ensayos cientificos. (Alianza Editorial: Madrid).

HICKMAN, F.R., 1986. Mathematical modelling in phisics, Physics Education, 21, pp. 173-180.

MARTINAND, J.L., 1986. Enseñanza y Aprendizaje de la modelización, Enseñanza de las Ciencias, 4, pp. 45-50.

MEHEUT, M. y LARCHER, C., 1987. Modèles particulaires dans l'initiation aux sciences physiques. Enseñanza de las Ciencias, Número Extra, pp. 261-262.

MORALES, J.F., 1987. Metodología y Teoría de la Psicología. (UNED: Madrid).
ROSADO, L., 1979. Didáctica de la Física. (Edelvives: Zaragoza).

ROSADO, L., 1986. Modelos en los Procesos de la Ciencia y su contrastación. (UNED: Madrid).

ROSADO, L., 1987. Electrónica Física y Microelectrónica. (Paraninfo: Madrid).

SCHEIDER, W., 1986. Models in Physics and Engineering, The Physics Teacher, 24, pp. 488-491.

SOLBES, J., CALATAYUD, M., CLIMENT, J. y NAVA. RRO, J., 1987. Errores conceptuales en los modelos at6micos cuánticos, Enseñanza de las Ciencias, 5, pp. $189-$ 195.

WENHAN,E.J., DORLING, G.W., SNELL, J.A.N. y TAYLOR, B., 1972. Phyisics Concepts and models. (Addison-Wesley: London). 